\title{
Analyzing Credit Evolution for Credit-based Incentive Schemes in Wireless Mesh Networks
}

\author{
Patrick P.C. Lee ${ }^{+} \quad$ Hongying Liu* John C.S. Lui ${ }^{+}$ \\ ${ }^{+}$Department of Computer Science and Engineering \\ ${ }^{*}$ School of Mathematics and Systems Science \\ The Chinese University of Hong Kong \\ \{pclee,cslui\}@cse.cuhk.edu.hk
Beihang University, Beijing, China
liuhongying@buaa.edu.cn

\begin{abstract}
Incentive mechanisms are often needed in wireless mesh networks (WMNs) so as to encourage nodes to relay or forward packets for other nodes. However, there is a lack of understanding on the interactions between the incentive mechanisms and the underlying routing protocols, and whether the network can sustain such traffic workload in the long run. In this paper, we propose a general mathematical framework via stochastic difference equations to model the interaction of incentive mechanisms and routing protocols. A credit evolution model is provided. Based on it, we study the expected credit variation of each node and use the Gini's coefficient to quantify the credit disparity among nodes. We also introduce the concept of differentiated pricing in the incentive mechanism to achieve credit equality among nodes so the WMN is more robust to sustain the traffic workloads.
\end{abstract}

\section{Introduction}

Wireless mesh networks (WMNs) have been a topic of intense research in the last several years. Such networks consist of a set of wireless and mobile nodes that can self-configure and do not rely on an infrastructure to communicate. Typically, a source communicates with distant destinations using intermediate nodes as relays. When nodes in WMNs are not under the control of a single authority, cooperation among nodes are paramount so packets can be relayed to the destination. Since there are selfish users who simply want to maximize their own welfare (e.g., monopolize the bandwidth) or refuse to relay packets, researchers have proposed different incentive mechanisms to encourage nodes to collaborate. These mechanisms can be broadly divided in two types: (a) reputation-based schemes [11], [12] and, (b) credit-based schemes [7]-[10]. In reputation-based schemes, a node's reputation is measured by its neighbors, and selfishness is deterred by the threat of partial or total disconnection from the network. Due to packet collisions and interference, nodes cannot always reliably detect if a given node actually forwarded a packet as expected, so it is possible that cooperative nodes will be perceived as being selfish, and wrongfully trigger a retaliation by their neighbors. In credit-based schemes, nodes receive a payment every time they forward a packet, and credit can be used by these nodes to transmit their own packets. Compared with the reputation schemes, credits enable more flexible and fine-grained control.

Although credit-based schemes can encourage cooperation among nodes. But it does not imply that the WMN can sustain any traffic workload. To illustrate, let us consider a WMN in Fig. 1.1. Let $\alpha$ be the payment from the source node to a

\author{
(1) $2(3)(5$ \\ Fig. 1.1. An example to a WMN to illustrate credit imbalance
}

relaying node for forwarding a message. When $\alpha$ is small, nodes have no incentive to forward packets. If $\alpha$ is sufficient to cover the cost of forwarding messages, the node has incentive to forward messages. Note that node 3 is in the center of the network and it costs less credit to send data to other nodes and has more chances to earn credit since traffic of other nodes has to go through node 3 . Node 1 or 5 , on the other hand, has no chance to earn any credit. If the payment parameter $\alpha$ is not properly set, node $1,2,4$, and 5 will eventually run out of credit and cannot transmit their own packets. To summarize, even if an incentive mechanism can guarantee cooperation, it is possible that the WMN cannot sustain the traffic workload. Contributions: The purpose of this paper is not to advocate any incentive mechanism, but rather, present a general mathematical framework to analyze the sustainability of various incentive mechanisms and routing protocols. Using our framework, designers can understand the interaction of routing protocols and incentive mechanisms, as well as the sustainability of the WMN. We quantitatively investigate the impact of credit inequality of credit-based incentive schemes. We take Sprite [9] as an illustrative example, and illustrate how it interacts with a family of path-based routing protocols. We consider the influence of link loss probability on the incentive scheme, and derive the closed-form expressions of the expected credit variation for some specific network topologies and traffic distribution. We also propose a differentiated pricing to achieve credit-equality.

The outline of our work is as follows. In Sec.II, we present the background of path-based routing protocols and creditbased incentive mechanisms. In Sec.III, we introduce our mathematical framework to model the credit evolution. In Sec.IV, we introduce differentiated pricing on the incentive mechanism. Results on the performance evaluation are given in Sec.V. Related work and conclusion are given in Sec. VI.

\section{Background and Technical Preliminaries}

In this section, we provide the background for our work. We first state the mathematical model for WMNs, the traffic model, as well as a family of path-based routing protocols. 
To illustrate our framework, we consider the Sprite [9] incentive protocol. We like to emphasize that our mathematical framework is very general and can accommodate other routing and incentive protocols. In here, we simply use the path-based routing and Sprite to illustrate the utility of our analytical framework.

Network model. We model a WMN as a directed graph $\mathcal{G}=$ $(\mathcal{N}, \mathcal{E}), \mathcal{N}$ is the set of wireless nodes with $|\mathcal{N}|=N$ and $\mathcal{E}$ is the set of wireless links. A link $(i, j) \in \mathcal{E}$ from node $i$ to node $j$ denotes that node $j$ is within the transmission range of node $i$. If there is no link from $i$ to $j$, then the message from node $i$ has to be transmitted to another node, and this node has to forward the message, either directly or indirectly, to $j$. Let $\epsilon_{(i, j)}$ be the loss probability of link $(i, j)$. A message from $i$ to $j$ on $(i, j)$ is correctly received with probability $1-\epsilon_{(i, j)}$.

Let $r$ be a route, which is a sequence of ordered nodes or an non-empty subset of $\mathcal{E}$. All routes in $\mathcal{G}$ is represented by the set $\mathcal{R}$. Let $\mathcal{S}$ be the set of all traffic sources in the network. For a traffic source $s \in \mathcal{S}$, let $\mathcal{D}_{s}$ be the set of its destinations. For a given source $s$ and its destination $d \in \mathcal{D}_{s}$, we define $(s, d)$ as the flow between node $s$ and $d$. Each flow has a set of routes, which is denoted by $\mathcal{R}_{s d}$ and $\left|\mathcal{R}_{s d}\right|$ represents the number of routes for flow $(s, d)$. Let $A_{s d}^{r}(t)$ be the traffic for flow $(s, d)$ on route $r$ at time $t$, then the traffic of flow $(s, d)$ at time $t$ is $A_{s d}(t)=\sum_{r \in \mathcal{R}_{s d}} A_{s d}^{r}(t)$. The total traffic arrival for source $s$ at time $t$ is $A_{s}(t)=\sum_{d \in \mathcal{D}_{s}} A_{s d}(t)$.

Traffic model. The packet arrival process $\left\{A_{s}(t)\right\}_{t=1}^{\infty}$ are i.i.d. sequences of a random variables for all $s \in \mathcal{S}$. The arrival rate $\mathrm{E}\left[A_{s}(t)\right]$ of source $s$ is denote by $\lambda_{s}$, wherein the expected time between two consecutive arrivals is 1 (or a unit slot). The destination of a flow is chosen as follows: with probability $h_{s d}$, the packets generated by the source node will choose node $d$ as the destination. Obviously, we have $\sum_{d \in \mathcal{D}_{s}} h_{s d}=1$ for $s \in \mathcal{S}$. Unless we state otherwise, we assume the uniform traffic model, which means $\lambda_{s}=\lambda$ and $h_{s d}=\frac{1}{N-1}$ for all $s, d$. Shortest-path based routing. In this paper, we consider a family of shortest-path based routing protocols, where the quality of a path can be chosen by (a) hop count, or (b) expected transmission count (ETX) [5]. Hop count assumes all links are homogeneous (i.e., links have the same loss probability, and channel fading characteristics). Therefore, for the flow $(s, d)$, Shortest-path hop-count routing selects those paths with the smallest link distance between $s$ and $d$.

Under ETX [5], it allows links to be heterogeneous. ETX minimizes the expected total number of packet transmissions required to successfully deliver a packet to the destination. ETX of a link is based on the delivery ratio: number of transmissions needed to successfully deliver a single packet. ETX of a path is the sum of the ETX for each link in that path. In general, ETX is able to reflect per-link performance and affect the per-flow performance. For the ease of mathematical formulation, we only consider use the forward delivery ratio to calculate these ETX values.

Consider the WMN in Fig. 2.1 as an example, where the link loss probability are shown along the edge. Consider the flow $(1,4)$. Under the shortest-path hop-count routing, there is

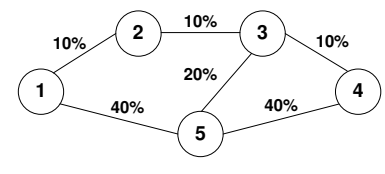

Fig. 2.1. Example of a WMN with link loss probabilities.

only one shortest path $(1,5,4)$. Under the shortest-path ETX routing, there are two shortest paths $(1,2,3,4)$ and $(1,5,4)$. Multi-path case. When flow $(s, d)$ has multiple shortest paths, or $\left|\mathcal{R}_{s d}\right|>1$, we use a traffic splitting approach. To illustrate, consider the flow $(1,4)$ in Fig. 2.1. Under the shortest-path ETX routing, there are two shortest paths $r=(1,2,3,4)$ and $r^{\prime}=(1,5,4)$. In this paper, we assume the uniform traffic splitting approach in our analysis, although our mathematical framework can accommodate more general splitting rule. This means $A_{14}^{r}(t)=A_{14}(t) /\left|\mathcal{R}_{14}\right|=A_{14}(t) / 2$.

Incentive scheme. Sprite [9] is a well-known incentive scheme to encourage nodes to collaborate. It has an important property of cheat-proof. Compared with other incentive schemes, Sprite does not require any tamper-proof hardware. Briefly speaking, it uses a centralize credit clearance service (CCS), to collect receipts from each forwarding nodes. Charges and rewards are based on these receipts, which provides incentive for nodes to cooperate and report actions honestly. Formally, Sprite can be described as follows:

(1) A sender selects a path to delivery a message to the destination. Denote this path as $r=\left(s, n_{1}, \cdots, n_{e}, \cdots, n_{l_{r}}\right)$, where $n_{l_{r}}=d$ and $l_{r}$ is the hop count of $r$.

(2) The sender will be charged for transmitting the message.

(3) CCS believes that a node along $r$ has forwarded the message if and only if there is a successor of that node on the path reporting a valid receipt of that message.

(4) CCS charges $C^{r}$ from node $s$, and pays $P_{n_{k}}^{r}$ to node $n_{k}$ :

$$
\begin{aligned}
C^{r}= & \left(l_{r}-1\right) \alpha+\beta-\left(l_{r}-e\right) \gamma \beta, \\
P_{n_{k}}^{r}= & \begin{cases}\alpha, & \text { if } k<e=l_{r} \\
\beta, & \text { if } k=e=l_{r} \\
\gamma \alpha, & \text { if } k<e<l_{r} \\
\gamma \beta, & \text { if } k=e<l_{r},\end{cases}
\end{aligned}
$$

here $n_{e}$ is the last node on path $r$ that submits a valid receipt, $\gamma<1$ and $\beta<\alpha$.

To illustrate, consider again the network in Fig. 2.1. We assume that node 1 is to send a message to node 4 along the path $r=(1,2,3,4)$, where $l_{r}=3$. When node 3 is the last node on path $r$ that submits a valid receipt, then $e=2, C^{r}=$ $2 \alpha+\beta-\gamma \beta, P_{2}^{r}=\gamma \alpha, P_{3}^{r}=\gamma \beta$ and $P_{4}^{r}=0$. When node 4 is the last node on path $r$ that submits a valid receipt, i.e. the message is send successfully, then $e=3, C^{r}=2 \alpha+\beta, P_{2}^{r}=$ $\alpha, P_{3}^{r}=\alpha$ and $P_{4}^{r}=\beta$.

Let $\delta$ be the cost of forwarding a receipt from one node to another. Then a colluding node (if any) incurs a cost of $\delta$ and $n_{k}$ must compensate the colluding node with $\delta$. We state some definitions and property of the Sprite system [9] here.

Definition 1: For a player, an optimal strategy is a strategy 


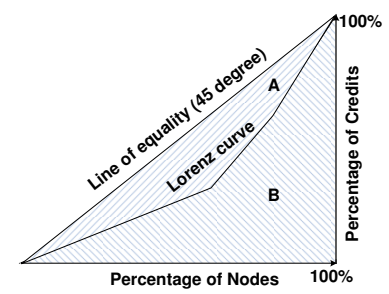

Fig. 2.2. A typical Lorenz curve for the network in Fig.2.1.

that brings the maximum expected welfare to it, regardless of the strategies of all the other nodes.

Definition 2: A game is collusion-resistant, if any group of colluding players cannot increase the expected sum of their welfare by using any strategy profile other than that in which everybody tells the truth.

Definition 3: A game is cheat-proof if truth-telling is an optimal strategy for all nodes and the game is collusion-resistant. Theorem 1: The receipt-submission game of Sprite, if $\delta \geq \gamma \beta$ and $\delta \geq\left(l_{r}-1\right) \gamma \alpha$, is cheat-proof.

Credit inequality metrics. In economics, the Lorenz curve [13] is a graphical representation of the cumulative distribution of wealth for a group of people. Here, we use it to represent credit distribution among nodes, where it shows for the poorest $x \%$ of nodes, what $y \%$ of the total credit of the system they possess. One can use the Lorenz curve to represent social (or credit) inequality. A Lorenz curve of a 45 degrees straight line thus represents perfect equality among nodes.

The Gini coefficient is defined based on the Lorenz curve, which is the ratio of the area that lies between the equality line and the Lorenz curve (marked 'A' in Fig. 2.2) over the total area under the equality line (marked ' $\mathrm{A}$ ' and ' $\mathrm{B}$ ' in Fig.2.2); i.e., $\mathrm{G}=\mathrm{A} /(\mathrm{A}+\mathrm{B})$. The Gini coefficient is a measure of the inequality of a distribution among nodes: $\mathrm{G}=0$ implies perfect equality and $\mathrm{G}=1$ implies maximal inequality.

To illustrate, consider again the network in Fig.2.1. Assume that there are total 10 credits. If each node has two credits, then the Lorenz curve is along the line of equality and $\mathrm{G}=$ 0 . If node 1,2 and 3 have one credit respectively, node 4 has three ones and node 5 has four ones, the Lorenz curve is shown in Fig.2.2 and $\mathrm{G}=0.21$.

\section{Mathematical Model For Credit Evolution}

Let us present the mathematical model to describe the credit evolution, i.e., the dynamic change of credit, for each node in a WMN. We model the evolution of nodes' credit as a sequence of random variables, and for some network topologies, we can obtain the closed form expression for the expected credit variation. We consider two scenarios:

(a) persistent transmission mode: when a collision or packet lost occurs, a node will retransmit the packet until it is successfully received by its neighbor.

(b) single transmission mode: when a collision or packet lost occurs, a node will not retransmit. The source node is responsible to retransmit the packet until the destination node receives the packet. Since source nodes need to perform retransmission, this can be regarded as the upper bound (or the worst case) payment for source nodes under Sprite [9]. For simplicity of presentation, we make the following assumptions:

- there is no cheating and no collusion for messageforwarding and receipt-submission,

- the link capacity is sufficient to satisfy all demands.

\section{A. General Mathematical Model}

We use $\mathcal{R}_{s d}^{n}=\left\{r \in \mathcal{R}_{s d}: n \in r\right\}$ to denote the route set (or path set) that node $n$ is an intermediate node of some route $r$ for flow $(s, d)$. Let $C^{r}$ be the cost for source node $s$ and $P_{n}^{r}$ be the payment to node $n \in r$ for successfully transmitting a packet along the path $r$ to $d$. Let $C_{n}(0)$ be the initial credit of node $n$. At time $t$, node $n$ has credit

$$
C_{n}(t)=C_{n}(0)+\sum_{k=1}^{t} \Delta C_{n}(k), \quad t \geq 1, n \in \mathcal{N},
$$

where

$$
\begin{aligned}
& \Delta C_{n}(k)=\sum_{s \in \mathcal{S}, d \in \mathcal{D}_{s}, r \in \mathcal{R}_{s d}^{n}} A_{s d}^{r}(k) P_{n}^{r} \\
& +\sum_{s \in \mathcal{S}, n \in \mathcal{D}_{s}} A_{s n}(k) \beta-\sum_{d \in \mathcal{D}_{n}, r \in \mathcal{R}_{n d}} A_{n d}^{r}(k) C^{r}
\end{aligned}
$$

is the credit variation of node $n$ in time slot $k$. In (3.2), the first and the second terms represent the payments from CCS to node $n$ for forwarding messages and for reporting receipts for the message respectively. The third term represents the payment from node $n$ to CCS for sending messages to other nodes. The credit evolution for the CCS is

$$
C(t)=C(t-1)-\sum_{n \in \mathcal{N}} \Delta C_{n}(t), \quad t \geq 1 .
$$

Persistent Transmission Mode: Under the persistent transmission mode, each intermediate node in the path would forward and/or retransmit an arbitrary number of times until a successful transmission occurs. In this case, we have $P_{n}^{r} \equiv \alpha$ for all $n \in r$ and $C^{r}=\left(l_{r}-1\right) \alpha+\beta$ for all $r \in \mathcal{R}$. Substituting them into equation (3.2), we have

$$
\Delta C_{n}(k)=B_{n}(k) \beta+F_{n n}(k) \alpha-\sum_{m \neq n} F_{n m}(k) \alpha,
$$

where $B_{n}(k)$ is the difference between the traffic ending at node $n$ and the one starting from node $n, F_{n n}(k)$ is the traffic that node $n$ forwards for others nodes, $F_{n m}(k)$ is the traffic that node $m$ forwards for node $n$ at time slot $k$. We have

$$
\begin{aligned}
B_{n}(k) & =\sum_{s \in \mathcal{S}, n \in \mathcal{D}_{s}} A_{s n}(k)-\sum_{d \in \mathcal{D}_{n}} A_{n d}(k) \\
F_{n n}(k) & =\sum_{s \in \mathcal{S}, d \in \mathcal{D}_{s}, r \in \mathcal{R}_{s d}^{n}} A_{s d}^{r}(k) \\
F_{n m}(k) & =\sum_{d \in \mathcal{D}_{n}, r \in \mathcal{R}_{n d}^{m}} A_{n d}^{r}(k) .
\end{aligned}
$$

Under the persistent transmission mode, the credit of CCS is always zero, i.e., $C(t) \equiv 0, t \geq 0$, which means that the credit variation of nodes is conservation, i.e., $\sum_{n \in \mathcal{N}} \Delta C_{n}(t)=0$ for $t \geq 1$.

Under the assumptions we made about the traffic model, $\left\{B_{n}(k)\right\}_{k=1}^{\infty}$ and $\left\{F_{n m}(k)\right\}_{k=1}^{\infty}$ are i.i.d. sequences of a random variables for all $n, m \in \mathcal{N}$ and $n \neq m$. By (3.3), the 
expected credit variation of node $n$ is

$$
\Delta c_{n}=b_{n} \beta+f_{n n} \alpha-\sum_{m \neq n} f_{n m} \alpha,
$$

where $\Delta c_{n}=\mathrm{E}\left[\Delta C_{n}(k)\right], b_{n}=\mathrm{E}\left[B_{n}(k)\right], f_{n m}=\mathrm{E}\left[F_{n m}(k)\right]$ and $f_{n n}=\sum_{s \neq n} f_{s n}$. Furthermore, by (3.1), the expected credit balance of node $n$ is

$$
\mathrm{E}\left[C_{n}(t)\right]=C_{n}(0)+t \Delta c_{n}, t \geq 1, n \in \mathcal{N} .
$$

Single Transmission Mode: Under this mode, intermediate nodes along the path will only forward the packet only once. In this case, $P_{n}^{r}$ and $C^{r}$ in equation (3.2) are random variables.

Assume $r=\left(s, n_{1}, \cdots, n_{l}\right)$ be a path for flow $(s, d)$, where $n_{l}=d$ and we omit the subscript $r$ from $l_{r}$. Let $\mathrm{A}_{i}$ denote the outcome that the message arrives at node $n_{i}$ but fails to arrive at node $n_{i+1}, i=1, \cdots, l$. Denote the probability that $\mathrm{A}_{i}$ happens by $p_{i}$. The corresponding cost of node $s$ and payment to intermediate node $n_{k}$ for outcome $\mathrm{A}_{i}$ are denoted by $c_{i}$ and $p_{i}^{n_{k}}$ for $k, i=1, \cdots, l$, respectively. Note that if the packet is not sent to node $n_{1}$, the CCS will not make an effective record. So we assume the source node $s$ always makes a successful transmission when we calculate the cost and payments.

Theorem 2: It holds that

(i) the expected cost that node $s$ successfully sends a message along path $r$ to node $d$ is $\bar{c} / p_{l}$, where $\bar{c}=$ $c_{1} p_{1}+\cdots+c_{l} p_{l}$, and

(ii) the expected payment to node $n_{k}$ that node $s$ successfully sends a message along path $r$ to node $d$ is $\bar{p}_{n_{k}} / p_{l}, k=1, \cdots, l$, where $\bar{p}_{n_{k}}=p_{1}^{n_{k}} p_{1}+\cdots+p_{l}^{n_{k}} p_{l}$.

Please refer to [18] for the proofs of Theorem 2 and other theorems hereafter.

We can further provide a closed-form expressions for $\mathrm{E}\left[C^{r}\right]$ and $\mathrm{E}\left[P_{n_{k}}^{r}\right], k=1, \cdots, l$. By the payoff scheme of Sprite (2.1) and (2.2), we have $c_{i}=(l-1) \alpha+\beta-(l-i) \gamma \beta$ and

$$
p_{i}^{n_{k}}= \begin{cases}\gamma \alpha, & \text { if } k<i \\ \gamma \beta, & \text { if } k=i \\ 0, & \text { if } k>i .\end{cases}
$$

Submitting them into the results in Theorem 2, we have $\mathrm{E}\left[C^{r}\right]=\frac{c_{l}-\gamma \beta \sum_{i=1}^{l-1}(l-i) p_{i}}{p_{l}}, \mathrm{E}\left[P_{d}^{r}\right]=\beta$ and $\mathrm{E}\left[P_{n_{k}}^{r}\right]=\alpha+$ $\gamma \frac{p_{k} \beta+\sum_{i=k+1}^{l-1} p_{i} \alpha}{p_{l}}, k=1, \cdots, l-1$, where $p_{i}=\prod_{j=1}^{i-1}(1-$ $\left.\epsilon_{\left(n_{j}, n_{j+1}\right)}\right) \epsilon_{\left(n_{i}, n_{i+1}\right)}, i=1, \cdots, l-1, p_{l}=\prod_{j=1}^{l-1}(1-$ $\left.\epsilon_{\left(n_{j}, n_{j+1}\right)}\right)$.

Remark: Let us provide a physical interpretation of Theorem 2. $1 / p_{l}$ is the expected number of transmissions that node $s$ makes for a message it sends along path $r$ to $d, \bar{c}$ is the expected cost that $s$ sends a message to $d$ and $\bar{p}_{n_{k}}$ is the expected payments to node $n_{k}, k=1, \cdots, l$. Therefore, the expected cost for $s$ to successfully send a message to $d$ is the product of the expected number of transmissions and the expected cost according to item (i) in Theorem 2. The similar results also hold for the payments to nodes $n_{1}, \cdots, n_{l}$.

Consider again the flow $(1,4)$ in Fig. 2.1, where node 1 sends a message along path $r=(1,2,3,4)$ to node 4 . We

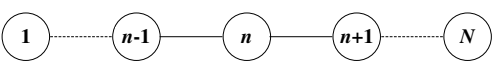

Fig. 3.1. Linear topology with $N$ nodes.

have $\mathrm{E}\left[C^{r}\right]=(100(2 \alpha+\beta)-29 \gamma \beta) / 81, \mathrm{E}\left[P_{2}^{r}\right]=(81 \alpha+$ $9 \gamma \alpha+10 \gamma \beta) / 81, \mathrm{E}\left[P_{3}^{r}\right]=(9 \alpha+\gamma \beta) / 9$ and $\mathrm{E}\left[P_{4}^{r}\right]=\beta$.

\section{B. Expected Credit Variation for Special Topologies with the Shortest Hop-count Routing}

We consider the expected credit variation for three special topologies with the shortest hop-count routing. Closed-form expressions can be given, where the expected traffic starting from $n$ is consistent with that ending at $n$, i.e. $\lambda$. So we have $b_{n}=0$ for all $n$. Let $F_{s n}(t)=\sum_{d \in \mathcal{D}_{s}, r \in \mathcal{R}_{s d}^{n}} A_{s d}^{r}(t)$ denote the traffic of $n$ forwarding for $s, s \neq n$.

Linear topology. We consider the one in Fig. 3.1. There is only one shortest path for each flow $(s, d)$. We have $F_{s 1}(t)=0$ and $F_{s N}(t)=0$. For $1<n<N$, we have

$$
\begin{gathered}
F_{s n}(t)= \begin{cases}A_{s d}(t), & \text { if } d>n, s<n \\
0, & \text { if } d<n, s<n\end{cases} \\
F_{s n}(t)= \begin{cases}0, & \text { if } d>n, s>n \\
A_{s d}(t), & \text { if } d<n, s>n .\end{cases}
\end{gathered}
$$

For the uniform traffic, by (3.5) we have that

$$
\begin{aligned}
f_{s n} & =\mathrm{E}\left[F_{s n}(t)\right]=\mathrm{E}\left[\mathrm{E}\left[F_{s n}(t) \mid d\right]\right] \\
& =\operatorname{Pr}(d>n) \mathrm{E}\left[A_{s d}(t)\right]=\frac{N-n}{N-1} \lambda
\end{aligned}
$$

if $s<n$. Similarly, by (3.6) we have $f_{s n}=\frac{n-1}{N-1} \lambda$ if $s>n$. Furthermore, we have

$$
\begin{aligned}
f_{n n} & =\left(\sum_{s=1}^{n-1} \frac{N-n}{N-1}+\sum_{s=n+1}^{N} \frac{n-1}{N-1}\right) \lambda \\
& =\frac{2(n-1)(N-n)}{N-1} \lambda
\end{aligned}
$$

and

$$
\begin{aligned}
\sum_{m: m \neq n} f_{n m} & =\left(\sum_{m=1}^{n-1} \frac{m-1}{N-1}+\sum_{m=n+1}^{N} \frac{N-m}{N-1}\right) \lambda \\
& =\frac{(n-2)(n-1)+(N-n-1)(N-n)}{2(N-1)} \lambda .
\end{aligned}
$$

Submitting them into equation (3.4), we have

$$
\Delta c_{n}=\frac{3 n(N-(n-1))-\frac{1}{2} N(N+3)-1}{N-1} \lambda \alpha,
$$

which is symmetric about $N / 2$.

Ring topology. Consider the WMN in Fig.3.2. For $n \in \mathcal{N}$, we let $s=\bmod (n \mp k, N), k=1,2, \cdots,\lfloor N / 2\rfloor$, where $\bmod$ is the modulo operation and $\lfloor N / 2\rfloor$ means the largest integer not greater than $N / 2$. In the sequel, we assume $\bmod (0, N)=$ $\bmod (N, N)=N$ for convenience of formulation.

We first consider the case that $N$ is odd, where each flow has only one shortest path. We have

$$
F_{s n}(t)= \begin{cases}A_{s d}(t), & \text { if } d=\bmod (n \pm l, N), \\ & \quad l=1, \cdots,\lfloor N / 2\rfloor-k \\ 0, & \text { otherwise. }\end{cases}
$$




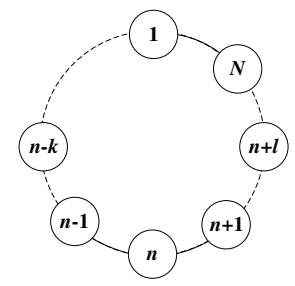

Fig. 3.2. Ring topology with $N$ nodes.

For the uniform traffic, similarly with the linear topology, by (3.7) we have $f_{s n}=\frac{\lfloor N / 2\rfloor-k}{N-1} \lambda$. Furthermore, we have

$$
\begin{aligned}
f_{n n} & =\sum_{s \neq n} f_{s n}=\sum_{m \neq n} f_{n m} \\
& =2 \sum_{k=1}^{\lfloor N / 2\rfloor} \frac{\lfloor N / 2\rfloor-k}{N-1} \lambda=\frac{(\lfloor N / 2\rfloor-1)\lfloor N / 2\rfloor}{N-1} \lambda .
\end{aligned}
$$

Submitting them into equation (3.4), we have $\Delta c_{n}=0$.

When $N$ is even, the flow $(s, \bmod (s+N / 2, N))$ has two shortest paths for all $s$. We have

$$
F_{s n}(t)= \begin{cases}A_{s d}(t), & \text { if } d=\bmod (n \pm l, N), \\ \quad l=1, \cdots, N / 2-k-1 \\ A_{s d}(t) / 2, & \text { if } d=\bmod (n \mp k+N / 2, N) \\ 0, & \text { otherwise. }\end{cases}
$$

For the uniform traffic, by equation (3.8) we have

$$
f_{s n}=\frac{N / 2-k-1}{N-1} \lambda+\frac{1}{N-1} \frac{\lambda}{2}=\frac{N / 2-k-1 / 2}{N-1} \lambda .
$$

Furthermore, we have

$$
\begin{aligned}
\sum_{m \neq n} f_{n m} & =f_{n n} \\
& =2 \sum_{k=1}^{N / 2-1} \frac{N / 2-k-1 / 2}{N-1} \lambda=\frac{(N / 2-1)^{2}}{N-1} \lambda .
\end{aligned}
$$

Submitting them into equation (3.4), we have $\Delta c_{n}=0$.

Grid topology. Consider the topology in Fig. 3.3, there is $N=H P$ nodes. In the sequel, let $n=p H+h$, where $p=$ $0,1, \cdots, P-1, h=1,2, \cdots, H$. For a given flow $(s, d)$, let $s=p_{s} H+h_{s}$ and $d=p_{d} H+h_{d}$. To obtain the credit variation of node $n$, we must know how much traffic it forwards for a given flow $(s, d)$, which needs the number of shortest path for flow $(s, d)$.

Lemma 1: The fact holds that $\left|\mathcal{R}_{s d}\right|=\left(\begin{array}{c}P_{0}+H_{0} \\ P_{0}\end{array}\right)$ for flow $(s, d)$, where $\left(\begin{array}{l}n \\ k\end{array}\right)$ is the binomial coefficient, $P_{0}=\left|p_{s}-p_{d}\right|$ and $H_{0}=\left|h_{s}-h_{d}\right|$ be the vertical and horizontal hop count between node $s$ and $d$, respectively.

In the sequel, we let $k_{1}=\left(\begin{array}{c}\left|p_{s}-p\right|+\left|h_{s}-h\right| \\ \left|p_{s}-p\right|\end{array}\right), \quad k_{2}=$ $\left(\begin{array}{c}\left|p-p_{d}\right|+\left|h-d_{d}\right| \\ \left|p-p_{d}\right|\end{array}\right), k=\left(\begin{array}{c}\left|p_{s}-p_{d}\right|+\left|h_{s}-h_{d}\right| \\ \left|p_{s}-p_{d}\right|\end{array}\right)$ and use the notation $\left(\begin{array}{l}0 \\ 0\end{array}\right)$ to denote 0 .

Lemma 2: If node $n$ forwards traffic for flow $(s, d)$, i.e. $\mathcal{R}_{s d}^{n} \neq \emptyset$, then $\sum_{r \in \mathcal{R}_{s d}^{n}} A_{s d}^{r}(t)=\frac{k_{1} k_{2}}{k} A_{s d}(t)$.

To get the closed-form of the expected credit variation of node $n$, three cases are consider respectively.

Case A: $p_{s}<p$. If $h_{s}<h$, we have

$$
F_{s n}(t)= \begin{cases}\sum_{r \in \mathcal{R}_{s d}^{n}} A_{s d}^{r}(t), & \text { if } p_{d}=p, \cdots, P-1, \\ & h_{d}=h, \cdots, H, d \neq n \\ 0, & \text { otherwise }\end{cases}
$$

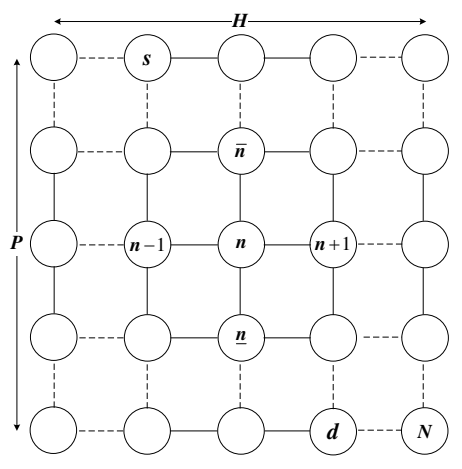

Fig. 3.3. Grid topology with $N=H P$ nodes.

Then the expected value of $F_{s n}(t)$ is

$f_{s n}=\sum_{p_{d}=p}^{P-1} \sum_{h_{d}=h}^{H} \operatorname{Pr}\left(d=p_{d} H+h_{d}, d \neq n\right) \mathrm{E}\left[\sum_{r \in \mathcal{R}_{s d}^{n}} A_{s d}^{r}(t)\right]$.

For the uniform traffic and Lemma 2, we have $f_{s n}=$ $k_{1} \sum_{p_{d}=p}^{P-1} \sum_{h_{d}=h}^{H} \frac{1}{N-1} \frac{k_{2}}{k} \lambda$. If $h_{s}=h$, we have $k_{1}=1$. Making a similar ananlysis with above, we have $f_{s n}=$ $\sum_{p_{d}=p}^{P-1} \sum_{h_{d}=1}^{H} \frac{1}{N-1} \frac{k_{2}}{k} \lambda$. If $h_{s}>h$, we have $f_{s n}=$ $k_{1} \sum_{p_{d}=p}^{P} \sum_{h_{d}=1}^{h} \frac{1}{N-1} \frac{k_{2}}{k} \lambda$.

Case B: $p_{s}=p$. Then $k_{1}=1$ and we have

$$
f_{s n}= \begin{cases}\sum_{p_{d}=0}^{P-1} \sum_{h_{d}=h}^{H} \frac{1}{N-1} \frac{k_{2}}{k} \lambda, & h_{s}<h \\ \sum_{p_{d}=0}^{P-1} \sum_{h_{d}=1}^{h} \frac{1}{N-1} \frac{k_{2}}{k} \lambda, & h_{s}>h .\end{cases}
$$

Case C: $p_{s}>p$. Then we have

$$
f_{s n}=\left\{\begin{array}{rr}
k_{1} \sum_{p_{d}=0}^{p} \sum_{h_{d}=h}^{H} \frac{1}{N-1} \frac{k_{2}}{k} \lambda, & h_{s}<h \\
\sum_{p_{d}=0}^{p} \sum_{h_{d}=1}^{H} \frac{1}{N-1} \frac{k_{2}}{k} \lambda, & h_{s}=h \\
k_{1} \sum_{p_{d}=0}^{p} \sum_{h_{d}=1}^{h} \frac{1}{N-1} \frac{k_{2}}{k} \lambda, & h_{s}>h .
\end{array}\right.
$$

Submitting $f_{s n}$ into equation (3.4), we can get the desired result.

\section{Balancing Credit via Pricing}

When a node has abundant amount of credit, this node has no incentive to forward packets for other nodes. On the other hand, when a node does not possess sufficient credit, it cannot transmit packet to its target receiver, so it needs to wait until it accumulates sufficient credits. These are undesirable since they reduce collaboration and lower system throughput. We address this problem here by determining the optimal incentive price for each node so to ensure fair distribution of credits.

Tradeoff between Incentive and Balancing: We introduce differentiated pricing by allowing different node to charge differently in forwarding a message. Under the Sprite mechanism, all nodes use the same price and we called this the homogeneous Sprite (HomoSprite). We consider the heterogeneous Sprite (HeteroSprite). Assume that node $s$ sends a message to $d$ along the path $r=\left(s, n_{1}, \cdots, n_{l_{r}}\right)$, where $n_{l_{r}}=d$ and $l_{r}$ 
is the hop count of path $r$. In HeteroSprite, the CCS charges $C^{r}$ from node $s$, and pays $P_{n_{k}}^{r}$ to node $n_{k}$, where

$$
\begin{aligned}
C^{r} & =\sum_{k=1}^{l_{r}-1} \alpha_{n_{k}}+\beta-\left(l_{r}-e\right) \gamma \beta \\
P_{n_{k}}^{r} & = \begin{cases}\alpha_{n_{k}}, & \text { if } k<e=l_{r} \\
\beta, & \text { if } k=e=l_{r} \\
\gamma \alpha_{n_{k}}, & \text { if } k<e<l_{r} \\
\gamma \beta, & \text { if } k=e<l_{r},\end{cases}
\end{aligned}
$$

where $n_{e}$ is the last node on path $r$ that submits a valid receipt, $\gamma<1$ and $\beta<\alpha_{n}$ for all $n$. Similar to [9], HeteroSprite has the following property:

Theorem 3: The receipt-submission game of HeteroSprite, if $\delta \geq \gamma \beta$ and $\delta \geq \gamma \sum_{k=1}^{l_{r}} \alpha_{n_{k}}$, is cheat-proof.

Comparing the expected gain of credit from forwarding a message with that of not forwarding the message, an intermediate node $m$ can expect a net gain of

$$
p_{2}(1-\gamma) \alpha_{m}+p_{1} \gamma\left(\alpha_{m}-\beta\right)
$$

where $p_{1}$ and $p_{2}$ are the probabilities that the message arrives at the next node and destination respectively. For the persistent transmission mode, similarly to (3.4) for HomoSprite, the expected credit variation of node $n$ for HeteroSprite is

$$
\Delta c_{n}=f_{n n} \alpha_{n}-\sum_{m \neq n} f_{n m} \alpha_{m}+w_{n},
$$

where $w_{n}=b_{n} \beta$ is independent of other nodes and

$$
f_{n n}=\sum_{s \neq n} f_{s n} .
$$

We need to consider two desirable requirements: one is to keep the expected credit variation (4.2) for each node as zero, i.e. $\Delta \mathbf{c}=\left(\Delta c_{1}, \cdots, \Delta c_{N}\right)=\mathbf{0}$. The other is to maximize the incentive to each node, i.e. maximize $\boldsymbol{\alpha}=\left(\alpha_{1}, \cdots, \alpha_{N}\right)$ according to (4.1). Therefore, a trade-off must be made between the two requirements. Taking $\|\Delta \mathbf{c}\|$ as the metric of the expected credit variation vector $\Delta \mathbf{c}$, we minimize $\|\Delta \mathbf{c}\|$ over the price vector $\boldsymbol{\alpha}$ subject to the box constraints, i.e.

$$
\begin{aligned}
& \operatorname{minimize}\|\Delta \mathbf{c}\| \\
& \text { subject to } \underline{\alpha} \leq \alpha_{n} \leq \bar{\alpha}, n=1, \cdots, N,
\end{aligned}
$$

where $\underline{\alpha}$ and $\bar{\alpha}$ is the lower and upper bound of the price, respectively. Note that we need $\underline{\alpha}>\beta$. Furthermore, it should be chosen such that the expected net gain of node $m$ in (4.1) be greater than the cost for forwarding a message. The upper bound $\bar{\alpha}$ should be less that $\frac{\delta}{h_{\max } \gamma}$, where $h_{\max }$ is the maximum hop count for possible routing. Then the price vector satisfies the condition established in Theorem 3.

To see the effect of the credit balancing method, we have to answer a more fundamental question: given an optimal price vector resulting from (4.4), what is the underlying physical meaning and how does it depend on the traffic workload of the system? We answer the problem via the shadow prices associated with the price constraints.
In constrained optimization, the shadow price is the change in the objective value of the optimal solution of an optimization problem obtained by relaxing the constraint by one unit: it is the marginal utility of relaxing the constraint, or equivalently the marginal cost of strengthening the constraint. Each constraint in an optimization problem has a shadow price or dual variable. The value of the shadow price can provide decision makers powerful insight into problem. In the sequel, we consider the shadow price for constraints (4.4b).

Taxicab Norm Pricing Approach: We take taxicab norm in (4.4a) and call it as taxicab norm pricing scheme. Introducing the nonnegative variable $x_{n}$, we translate the associated nonsmooth optimization problem (4.4) to a linear programming problem

$$
\begin{array}{ll}
\text { minimize } & \sum_{n=1}^{N} x_{n} \\
\text { subject to } & f_{n n} \alpha_{n}-\sum_{m \neq n} f_{n m} \alpha_{m}+x_{n} \geq-w_{n} \\
& -f_{n n} \alpha_{n}+\sum_{m \neq n} f_{n m} \alpha_{m}+x_{n} \geq w_{n} \\
& \underline{\alpha} \leq \alpha_{n} \leq \bar{\alpha}, n=1, \cdots, N .
\end{array}
$$

The dual of the optimization problem (4.5) is given by

$$
\begin{aligned}
\operatorname{maximize} & \sum_{n=1}^{N}\left(w_{n} z_{n}-w_{n} y_{n}+\underline{\alpha} \mu_{n}-\bar{\alpha} \nu_{n}\right) \\
\text { subject to } & \sum_{s \neq n} f_{s n}\left(z_{s}-y_{s}\right) \\
& -f_{n n}\left(z_{n}-y_{n}\right)+\mu_{n}-\nu_{n}=0 \\
& y_{n}+z_{n}=1 \\
& y_{n}, z_{n}, \mu_{n}, \nu_{n} \geq 0, n=1, \cdots, N .
\end{aligned}
$$

Let $\left(\boldsymbol{\alpha}^{*}, \mathbf{x}^{*}\right)$ be the solution to (4.5). Then the expected credit variation of node $n$ is $\Delta c_{n}^{*}=f_{n n} \alpha_{n}^{*}-\sum_{m: m \neq n} f_{n m} \alpha_{m}^{*}+w_{n}$. It can be easily check that $x_{n}^{*}=\left|\Delta c_{n}^{*}\right|$ for all $n$.

Let $R=\left\{n \in \mathcal{N}: \Delta c_{n}^{*}=x_{n}^{*}\right\}$ be the index set of rich node, i.e., whose expected credit variation is positive, and $P=$ $\left\{n \in \mathcal{N}: \Delta c_{n}^{*}=-x_{n}^{*}\right\}$ is the index set of poor node, i.e., whose expected credit variation is negative. Define index sets $L=\left\{n \in \mathcal{N}: \alpha_{n}^{*}=\underline{\alpha}\right\}, M=\left\{n \in \mathcal{N}: \underline{\alpha}<\alpha_{n}^{*}<\bar{\alpha}\right\}$ and $U=\left\{n \in \mathcal{N}: \alpha_{n}^{*}=\bar{\alpha}\right\}$. The following theorem shows how the optimal price vector depends on the traffic workload.

Theorem 4: Let $\left(\boldsymbol{\alpha}^{*}, \mathbf{x}^{*}\right)$ be the solution of (4.5). Let $\left(\mathbf{y}^{*}, \mathbf{z}^{*}, \boldsymbol{\mu}^{*}, \boldsymbol{\nu}^{*}\right)$ be a solution of (4.6). Then

(i) $\forall n \in R \cap(U \cup M)$, it holds that $\mu_{n}^{*}=\nu_{n}^{*}=0$; In addition, $f_{\text {sn }}=0, \forall s \in P, s \neq n$.

(ii) $\forall n \in R \cap L$, it holds that $\mu_{n}^{*}=2 \sum_{s \in P, s \neq n} f_{s n}$ and $\nu_{n}^{*}=0$. (iii) $\forall n \in P \cap U$, it holds that $\nu_{n}^{*}=2 \sum_{s \in R, s \neq n} f_{s n}$ and $\mu_{n}^{*}=0$. (iv) $\forall n \in P \cap(M \cup L)$, it holds that $\mu_{n}^{*}=\nu_{n}^{*}=0$; In addition, $f_{s n}=0, \forall s \in R, s \neq n$.

Note that we can carry out similar analysis of the shadow price for the pricing method with infinity norm or even Euclidean norm in (4.4a). In view of complexity, both infinity norm and taxicab norm pricing approaches need to solve a linear programming problem. For Euclidean norm pricing approach, it is necessary to solve a linear least-squares problem with box constraints, which is also solvable in polynomial time.

\section{Performance Evaluation}

\section{A. Simulation Setup}

For the linear and ring topologies, we set $N=10$. For the grid topology, set $H=5$ and $P=5$, or $N=25$. A topology 


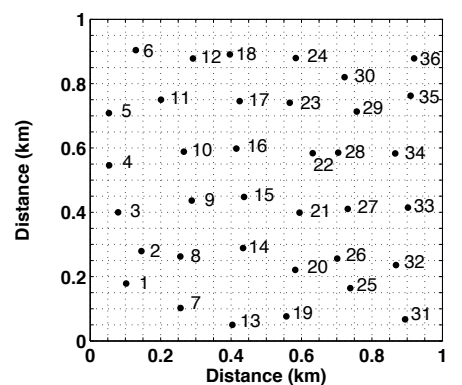

Fig. 5.1. Specific topology randomly generated.

consists of $N=36$ nodes that are randomly distributed in an area of 1000 by 1000 meters as shown in Fig. 5.1. We use the lognormal shadowing propagation model [4]. Let $d_{i j}$ and $p_{i j}$ be the distance and the delivery probability for the link from node $i$ to node $j$, respectively. Based on [4], $p_{i j}$ can be approximated as a function of $d_{i j}$ :

$$
p_{i j}= \begin{cases}1-\left(\frac{d_{i j}}{P}\right)^{2 \rho} / 2, & \text { if } d_{i j} \leq D \\ \left(\frac{2 D-d_{i j}}{D}\right)^{2 \rho} / 2, & \text { if } D<d_{i j} \leq 2 D \\ 0, & \text { otherwise, }\end{cases}
$$

where $\rho$ is the power attenuation factor ranging from 2 to 6 , and $D$ is defined as the distance such that $p_{i j}(D)=0.5$. In our simulation, we set $\rho=5$ and $D=200 \mathrm{~m}$. We assume $j$ is not in the transmission range of node $i$ if $p_{i j}<0.6$. The average degree of a vertex is 3.44 .

The source's traffic arrival rate is set as $\lambda=1$ under the uniform traffic model. In HomoSprite, we set the parameters $\alpha=1, \beta=0.1$ and $\gamma=0.001$. In the pricing model (4.4) for HeteroSprite, we set $\underline{\alpha}=0.5$ and $\bar{\alpha}=10$.

\section{B. Persistent Transmission Mode vs Single Transmission Mode}

In this experiment, a comparison is made for HomoSprite respectively under the persistent transmission mode and the single one. The specific topology shown in Fig.5.1 is considered here. The expected credits variation and Lorenz curves with corresponding Gini coefficients are shown in Fig. 5.2.

One can observe from Fig. 5.2 that the expected credits variation of nodes under the single transmission mode is smaller than that under the persistent transmission mode.

The difference between the expected credit variation of the nodes on the edge of the network, e.g. node 1, 2, 3, 35 and 36 , under two transmission modes, are much larger than those in the center of the network.

The Lorenz curves in Fig. 5.2 (a) and (b) show that the expected credits variation under the persistent transmission mode is more equal than the one under the single transmission mode. The CCS has null credit under the persistent transmission mode. While under the single transmission mode, the CCS has 68 credits in the shortest hop-count routing and 55 credits in the shortest-ETX routing, where a unsolved problem for CCS under the single transmission mode is how to redistribute those surplus credits to each node. For the two said transmission
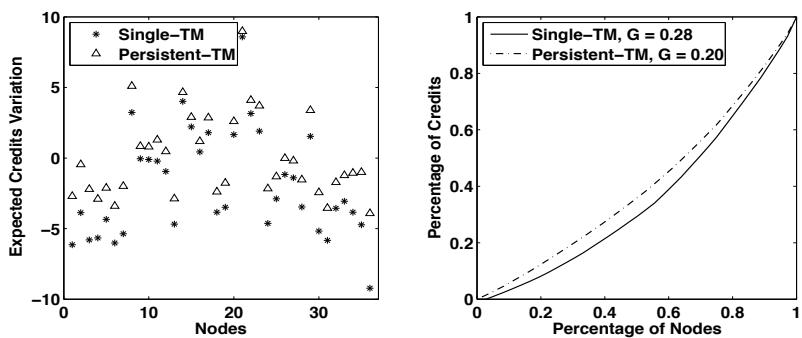

(a) Shortest-path hop-counting routing
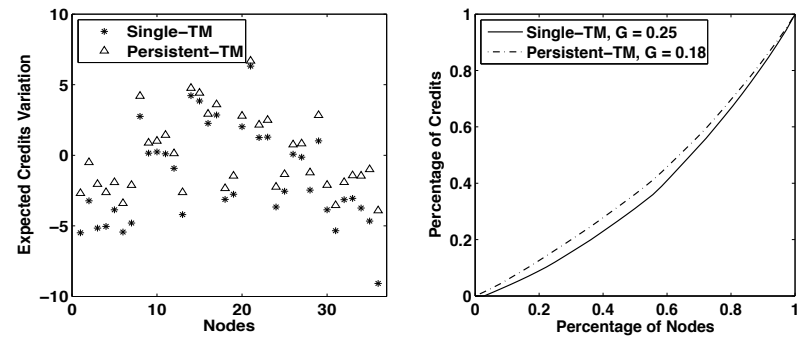

(b) Shortest-path ETX routing

Fig. 5.2. Persistent transmission mode against single transmission mode.

modes, ETX is more appropriate than hop count as a routing metric in evenly distributing credit among nodes.

\section{Credit Inequality Measures for Expected Credits Variation}

In the following experiments, we focus on HomoSprite under the persistent transmission mode. The expected credits variation and Lorenz curves for the linear, ring and grid topologies under different routing metrics and different traffic models are considered.

Experiment 1 Comparison between different routing metrics. Fig. 5.3 shows the expected credits variation and Lorentz curves for the linear and ring topologies under the hop count metric and the ETX metric, where the link loss probabilities are uniformly generated over $[0,0.4]$ for ETX metric.

For the linear topology, each flow has the same routing in the shortest path hop-count routing and the shortest ETX routing. The expected credits variation shown in Fig.5.3 (a) and (b) are the same under the two routing protocols, and nodes 1 and 10, which are on the edge of the network, have the smallest expected credits variation, while nodes 5 and 6 , which are in the center of the network, have the highest expected credits variation.

For the ring topology, as shown in Fig.5.3 (a), each node plays a same role and maintains credit equality under hop count metric. From Fig.5.3 (b), we observe that the shortest ETX routing has almost no effect on the credit distribution.

To test the effect of the ETX measure on the credit inequality in grid topology, we consider two scenarios for the shortestpath ETX routing. One is the ETX-Edge scenario, where the links out from the nodes on the edges of the network have zero loss probability and others 0.3 . The other is the ETXCross scenario, where the links out from the nodes on the middle cross have zero loss probability while others 0.3 . The 

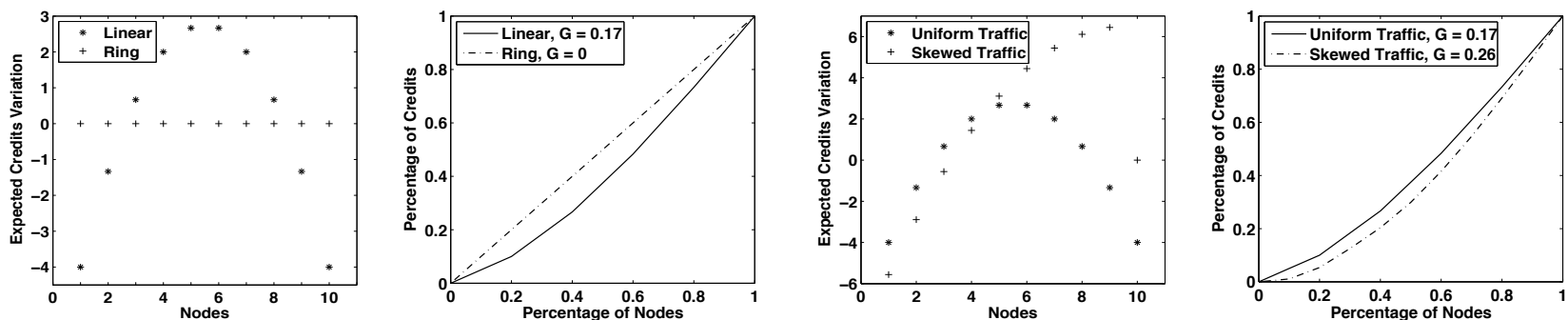

(a) Hop count metric
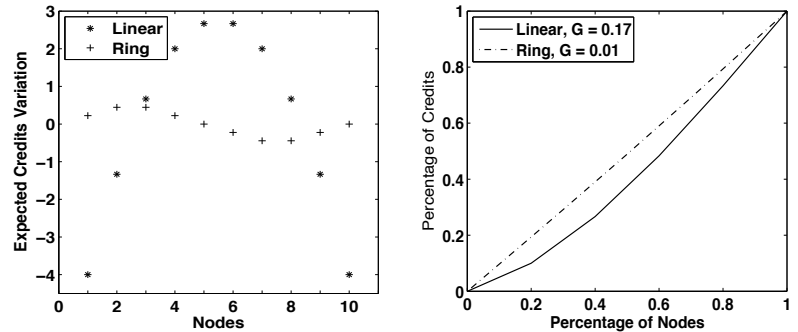

(b) ETX metric
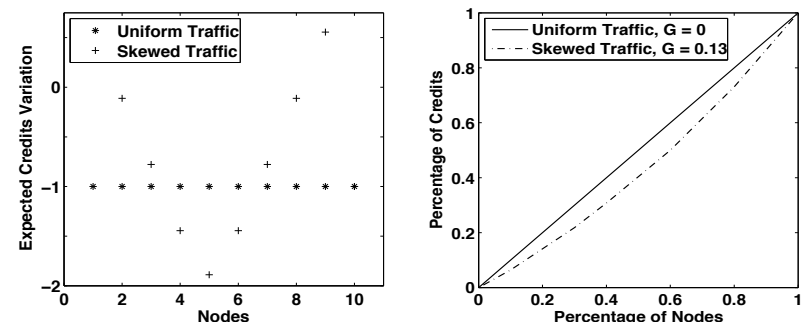

(b) Ring topology

Fig. 5.3. Hop count metric against ETX metric for linear and ring topologies.

results are shown in Fig.5.4. Compared with the shortest-path hop-count routing, the ETX-Edge case makes the credit more equal because there are more opportunities for nodes on the edge of the network to forward message for others. On the contrary, the ETX-Cross case has credit inequality since there are more opportunities for nodes in the center of the network to earn more credits.
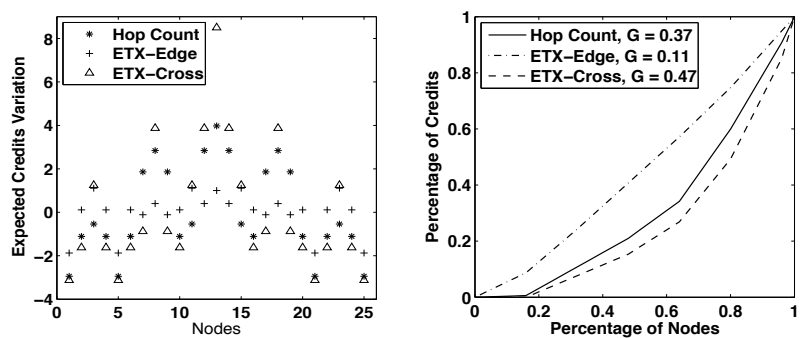

Fig. 5.4. Hop count metric against ETX metric for grid topology.

Experiment 2 Comparison between different traffic patterns. We consider a skewed traffic model, i.e., $\lambda_{N}=\lfloor N / 2\rfloor \lambda$ and $\lambda_{s}=\lambda$ for all $s \neq N$. In addition, $h_{N d}=\frac{1}{N-1}$ for all $d \neq N$, and for all $s \neq N$

$$
h_{s d}= \begin{cases}\frac{1}{2(N-2)} & d \neq s, N \\ \frac{1}{2} & d=N .\end{cases}
$$

This traffic model can be regarded as the one in a hybrid WMN with one access point $N$.

The expected credits variation and Lorenz curves with the shortest-path hop-count routing are shown in Fig.5.5. From Fig. 5.5 (a) and (b), one can conclude that the skewed traffic in linear and ring topologies will magnify the credit inequality.

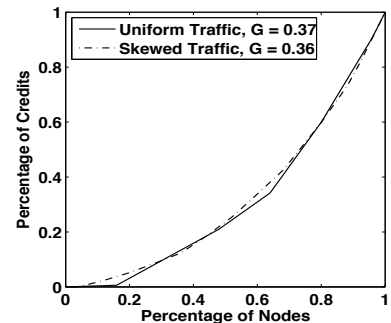

(c) Grid topology

Fig. 5.5. Uniform traffic against skewed traffic.

While skewed traffic in grid topology will slightly reduce the credit inequality because there are more chances for some nodes on the edge of the network to earn credits, as illustrated in Fig.5.5 (c).

\section{Effectiveness of Differentiated Pricing}

To illustrate the effectiveness of differentiated pricing, the uniform traffic model with the shortest-path ETX routing is considered for the topology in Fig.5.1.

The expected credits variation and Lorenz curves for HomoSprite and HeteroSprite are shown in Fig. 5.6. It can be seen that for the HeteroSprite, most nodes that have the chance to forward messages for others almost have zero expected credits variation. It implies that we can achieve credit equality.

We let $C_{n}(0)=\frac{100 K}{N}, n \in \mathcal{N}$, where $K$ is the sum of the expected cost of each node under the uniform traffic model. In each time slot, each source $s$ generates flow $(s, d)$ with traffic $A_{s d}(t)$, which is randomly chosen from the Poisson distribution with parameter 1 . If the credit balance of source $s, C_{s}(t)$, is greater than the cost of transmission traffic $A_{s d}(t)$, the sending succeeds and $C_{s}(t)$ and $C_{n}(t)$ for all $n \in \mathcal{R}_{s d}$ are updated according to the general model (3.1); Otherwise, the sending fails. We simultaneously run the two systems 500 time slots. The goodputs of HomoSprite and HeteroSprite are 

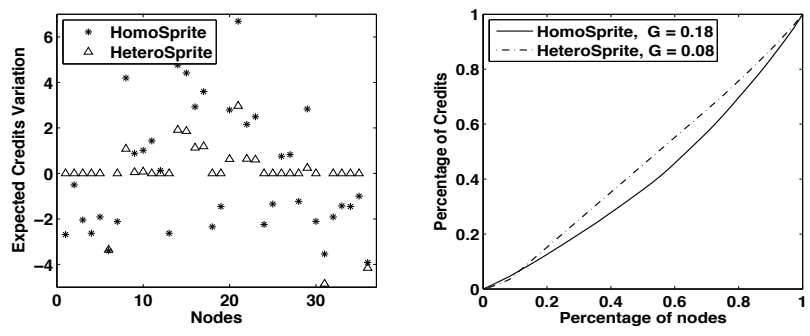

Fig. 5.6. The expected credits variation of HomoSprite and HeteroSprite.

shown in Fig.5.7. For a source, the goodput means the ratio of successfully sent messages to all generated ones.

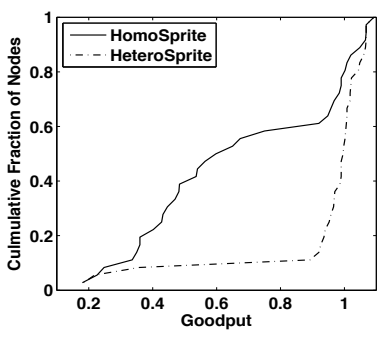

(a) $\mathrm{CDF}$ of Sprite goodput

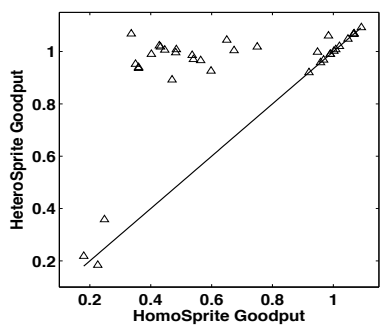

(b) Scatter plot of Sprite goodput
Fig. 5.7. Goodput of HomoSprite and HeteroSprite.

From Fig.5.7 (a), we observed that the goodput of almost half the nodes in HeteroSprite are 70 percent higher at the most than the one in HomoSprite. The scatter plot in Fig. 5.7 (b) shows more details. The fewer nodes that have no opportunities to forward message for others and nodes that have much opportunities to forward message for others keep the same goodputs in HomoSprite and HeteroSprite. The most nodes have a higher goodput in HeteroSprite than that in HomoSprite. In addition, the mean value of goodput for HomoSprite and HeteroSprite are 0.67 and 0.92 respectively. The median value of goodput for HomoSprite and HeteroSprite are 0.61 and 0.97 respectively. The standard deviation of goodput for HomoSprite and HeteroSprite are 0.29 and 0.21 respectively. All these facts indicate that nodes in HeteroSprite can achieve higher and more stable goodput than that in HomoSprite. It implies that the WMN with HeteroSprite as incentive scheme can runs more health and robust.

\section{Related Work and Conclusion}

From a game theory perspective, credit-based [7]-[10] and reputation-based [11], [12] incentive mechanisms in WMNs have been proven effective in motivating cooperation of nodes. For routing protocols in WMNs, Srcr [6] is a state-of-the-art path routing protocol, where link weights are assigned based on the ETX metric [5].

Simple models have been proposed to capture the distribution of money [13] in economics. There also exist models for study of the condensation of materials [14] in physics. Friedman et al. [15] study the credit-based P2P system and concludes that too large an mount of internal currency injected into the system will cause the system to collapse. Zhao et al. [16], [17] proposed a general analytical framework to analyze and design a large family of incentive protocols for $\mathrm{P} 2 \mathrm{P}$ networks. The main difference of incentive scheme in WMNs and $\mathrm{P} 2 \mathrm{P}$ networks is that the peers is not directly interacting with each other and we need to consider the interactions between the incentive mechanisms and the routing protocols.

In this paper, we present a mathematical framework to analyze the interaction of the credit-based incentive scheme (e.g., such as Sprite) and path-based routing protocols such as the shortest-path-hop-count or ETX [5]. We showed that under some traffic workload, the WMNs can have large credit-inequality, which can cause some nodes not able to transmit any packet. To redeem this problem, we propose a differentiated pricing mechanism so as to evenly distribute credit among nodes such that the Gini coefficient of the WMN is closed to zero (or achieving the credit equality). The mathematical methodology we propose also opens doors to investigate the sustainability of wireless networks that employ different incentive mechanisms and/or routing protocols.

Acknowledgement: The work of John C.S. Lui and Patrick Lee were supported in part by the RGC Grant 415309 and 413910 respectively.

\section{REFERENCES}

[1] Geoffrey R. Grimmett and David R. Stirzaker. Probability and Random Process (3rd ed.). Oxford University Press, 2001.

[2] Evans, Merran, Nicholas Hastings and Brian Peacock. Statistical Distributions (3rd ed.). Wiley, 2000, pp. 134-136.

[3] George B. Dantzig. Linear Programming and Extensions. Princeton University Press, 1963.

[4] The network simulator - ns-2. http://www.isi.edu/nsnam/ns/

[5] D. S. J. De Couto, D. Aguayo, J. Bicket and R. Morris. A high-throughput path metric for multi-hop wireless routing. In Proceedings of MobiCom'03, 2003.

[6] J. Bicket, D. Aguayo, S. Biswas, and Morris. Atchitecture and evaluation of an unplanned 802.11b mesh network. In Proceedings of MobiCom'05, 2005.

[7] L. Buttyan and J. P. Hubaux. Enforcing service availability in mobile ad-hoc WANs. In IEEE/ACM Workshop on Mobile Ad Hoc Networking and Computing (MobiHOC), Boston, MA, August 2000.

[8] L. Buttyan and J. P. Hubaux. Stimulating cooperation in self-organizing mobile ad hoc networks. ACM Journal for Mobile Networks \& Applications, Vol. 8, pp.579$592,2003$.

[9] S. Zhong, J. Chen and Y. R. Yang. Sprite, a simple, cheat-proof, credit-based system for mobile ad-hoc networks. IEEE INFOCOM, 2003.

[10] S. Zhong, L. Li, Y. Liu and Y. R. Yang. On designing incentive-compatible routing and forwarding protocols in wireless ad-hoc networks - an integrated approach using game theoretical and cryptographic techniques. In Proceedings of MobiCom'05, Cologne, Germany, Sept. 2005.

[11] S. Marti, T. Giuli, K. Lai and M. Baker. Mitigating routing misbehavior in mobile ad hoc networks. In Proceedings of MobiCom'00, Boston, USA, Aug. 2000.

[12] J. Jaramillo and R. Srikant. DARWIN: Distributed and adaptive reputation mechanism for wireless ad-hoc networks. In Proceedings of MobiCom'07, Montréal, Québec, Canada, Sept. 2007.

[13] R. Steckel and C. Moehling. Rising inequality: trends in the distribution of wealth in industrializing New England. The Journal of Economic History, 61(1), pp.160$183,2001$.

[14] J.-P. Bouchaud and M. Mézard. Wealth condensation in a simple model of economy. Physica A, vol. 282, no.3, pp.536-545, 2000.

[15] Eric J. Friedman, Joseph Y. Halpern and Ian Kash. Efficiency and equilibria in a scrip system for P2P networks. In Proceeding of the 7th ACM Conference on Electronic Commerce (EC'06), Ann Arbor, USA, Jun. 2006.

[16] B.Q. Zhao, John C. S. Lui and D.M. Chiu. Analysis of adaptive incentive protocols for P2P networks. IEEE INFOCOM 2009, Rio de Janeiro, Brazil.

[17] B.Q. Zhao, John C. S. Lui and D.M. Chiu. Mathematical Framework for Analyzing Adaptive Incentive Protocols in P2P Networks. To appear in IEEE/ACM Transactions on Networking.

[18] P. C. Lee, John C. S. Lui, Hongying Liu. Analyzing Credit Evolution for Credit-based Incentive Schemes in Wireless Mesh Networks. URL: www.cse.cuhk.edu.hk/ cslui/technical_report08.pdf 